12,13

\title{
Компьютерное моделирование структуры и механических свойств слоев силицена на графите при движении иона лития
}

\author{
(C) А.Е. Галашев, К.А. Иваничкина \\ Институт высокотемпературной электрохимии УрО РАН, \\ Екатеринбург, Россия \\ E-mail: galashev@ihte.uran.ru
}

(Поступила в Редакцию 28 мая 2018 г.)

\begin{abstract}
Методом молекулярной динамики исследованы структурные и механические эффекты, появляющиеся при движении иона лития в постоянном электрическом поле по плоскому каналу, образованному совершенными листами силицена и листами, содержащими дефекты вакансионного типа. Моно-, би-, три- и гексавакансии достаточно плотно и равномерно заполняли листы силицена, размещенные один над другим на графитовой подложке. Определены времена прохождения ионом $\mathrm{Li}^{+}$силиценовых каналов, имеющих различные зазоры. Построение многогранников Вороного и усеченных многогранников, центры которых совпадают с положением движущегося иона, позволило выявить структурные особенности, присущие двумерной слоистой структуре. Установлен характер появляющихся в листах силицена напряжений, наиболее критичных к перемещению иона по каналу.
\end{abstract}

DOI: $10.21883 /$ FTT.2019.02.47139.146

\section{1. Введение}

Обычные литий-ионные батареи на основе графитовых анодов уже не могут обеспечить удовлетворительную производительность, что включает большую емкость, высокую скорость, хорошую цикличность и низкую цену [1-3]. Двумерные (2D) материалы являются основными кандидатами для использования в качестве материала анода и привлекают большое внимание в последнее время из-за уникальных структурных свойств [4-6]. Дело в том, что двумерная структура может обеспечить большую площадь поверхности для размещения атомов лития. Кроме того, свободная упаковка между двумерными слоями может облегчить расширение и сжатие объема, вызванное интеркалированием и деинтеркаляцией атомов лития. Большинство синтезированных на сегодняшний день силиценов осаждаются на металлические подложки, такие как $\mathrm{Ag}$ (111) и $\operatorname{Ir}(111)$ [7,8]. Сильное взаимодействие между подложками и выращенным на них силиценом нарушает электронную структуру автономного силицена $[9,10]$ и способно индуцировать поверхностную реконструкцию [11]. Как следствие, теряются очень важные перспективные свойства автономного силицена, нужные для его применения в качестве анодного материала. Методом молекулярно-динамического моделирования было показано, что графен может служить в качестве подложки для силицена вместо металлических подложек [12,13]. В отличие от металлических подложек взаимодействие между силиценом и графеном в основном определяется силами Ван-дер-Ваальса, за счет чего могут быть сохранены уникальные свойства силицена. Однако в настоящее время применение графена для поддержки силицена можно рассматривать лишь как гипотетическую возможность. Технология изготовления такого нанокомпозита не разработана.

Квазинепрерывный слой двумерного кремния на подложке инертного высокоориентированного пиролитического графита (ВОПГ) при комнатной температуре в условиях сверхвысокого вакуума был получен в [14]. Было обнаружено присутствие небольшого количества участков ВОПГ, существующих вместе с образовавшимися небольшими 3D-кластерами Si. Данные сканирующей туннельной микроскопии свидетельствуют о наличии очень малой деформации $(0.05 \mathrm{~nm})$ силицена. Силы Ван-дер-Ваальса создают связь силицена с поверхностью. Наличие такого взаимодействия делает идеальный силиценовый монослой на поверхности ВОПГ устойчивым как при комнатной температуре, так и при $350^{\circ} \mathrm{C}$. Рост силицена на неметаллической инертной подложке является важным шагом на пути к получению кремниевых слоев, не содержащих сплавов и обладающих свойствами, близкими к автономному силицену. Силицен открывает перспективу полной совместимости его применения с уже существующими кремниевыми технологиями. Более того, силицен на графитовой подложке представляет собой гораздо менее дорогостоящее решение, чем технологии на основе серебряных подложек [15]. Исследования, представленные в работе [15], указывают на принципиальные трудности использования листов силицена на серебряных подложках как электрода литий-ионной батареи.

Цель настоящей работы - изучение возможности применения слоистого кремния в виде листов силицена в качестве материала анода литий-ионной батареи. Вычисления проводились с использованием кодов программного комплекса LAMMPS [16]. Расчеты выполнены на гибридном вычислителе кластерного типа „УРАН“ при ИММ УрО РАН. 


\section{2. Компьютерная модель}

Межатомные взаимодействия $\mathrm{Si}-\mathrm{Si}, \mathrm{Si}-\mathrm{C}$ и $\mathrm{C}-\mathrm{C}$ представлялись многочастичным потенциалом Терсоффa $[17,18]$

$$
V_{i j}=f_{C}\left(r_{i j}\right)\left[A \exp \left(-\lambda^{(1)} r_{i j}\right)-B b_{i j} \exp \left(-\lambda^{(2)} r_{i j}\right)\right],
$$$$
f_{C}\left(r_{i j}\right)= \begin{cases}1, & r_{i j}<R^{(1)} \\ \frac{1}{2}+\frac{1}{2} \cos \left[\frac{\pi\left(r_{i j}-R^{(1)}\right)}{\left(R^{(2)}-R^{(1)}\right)}\right], & R^{(1)}<r_{i j}<R^{(2)}, \\ 0, & r_{i j}>R^{(2)}\end{cases}
$$

$$
\begin{aligned}
& b_{i j}=\left(1+\beta^{n} \xi_{i j}^{n}\right)^{-1 /(2 n)}, \\
& \xi_{i j}=\sum_{k \neq i, j} f_{C}\left(r_{i j}\right) g\left(\theta_{i j k}\right),
\end{aligned}
$$$$
g\left(\theta_{i j k}\right)=1+\frac{c^{2}}{d^{2}}-\frac{c^{2}}{\left[d^{2}+\left(h-\cos \theta_{i j k}\right)\right]} .
$$

При взаимодействии атомов, принадлежащих компонентам $p$ и $q$, параметры $\lambda^{(1)}, \lambda^{(2)}, A, B, R^{(1)}$ и $R^{(2)}$ определяются как

$$
\begin{aligned}
\lambda^{(k)}= & \left(\lambda_{p}^{(k)}+\lambda_{q}^{(k)}\right) / 2 ; \quad A-\left(A_{p} A_{q}\right)^{1 / 2} ; \quad B=\left(B_{p} B_{q}\right)^{1 / 2} \\
& R^{(1)}=\left(R_{p}^{(1)} R_{q}^{(1)}\right)^{1 / 2} ; \quad R^{(2)}=\left(R_{p}^{(2)} R_{q}^{(2)}\right)^{1 / 2}
\end{aligned}
$$

Здесь параметры $A$ и $B$ являются энергетическими характеристиками отталкивания и притяжения; многочастичный параметр $b_{i j}$ зависит от локальной координации атомов вокруг атома $i$; имеющие размерность обратной длины параметры $\lambda^{(1)}$ и $\lambda^{(2)}$ задают степень отталкивания и притяжения соответственно; параметры $R^{(1)}$ и $R^{(2)}$ с размерностью длины были выбраны так, чтобы включать в рассмотрение только ближайших соседей; параметры $n, n_{i}$ и $\beta$ устанавливают действующую на атом силу связывания в зависимости от окружающей среды; $\xi_{i j}$ - эффективное координационное число; с помощью индексов $i, j, k$ обозначены атомы углерода, $r_{i j}$ - длина связи $i-j, \theta_{i j k}-$ угол между связями $i-j$ и $j-k ; g\left(\theta_{i j k}\right)$ - функция этого угла, стабилизирующая структуру; параметр $d$ задает ширину острого максимума в угловой зависимости $g(\theta)$, парамтр $c$ задает высоту этого пика, функция $g(\theta)$ имеет минимум при $h=\cos \theta$.

Параметры потенциала Терсоффа, определенные для случая $s p^{3}$-гибридизации, могут быть использованы для описания взаимодействия в материалах с $s p^{2}$-гибридизацией [19].

Взаимодействия $\mathrm{Si}-\mathrm{Si}$ между атомами, принадлежащими разным листам силицена, описывались потенциалом Морзе [20]

$$
\Phi(r)=D_{e}\left[\exp \left\{-2 \alpha\left(r-r_{e}\right)\right\}-2 \exp \left\{-\alpha\left(r-r_{e}\right)\right\}\right],
$$

где $D_{e}-$ глубина потенциальной ямы, $\alpha$ - параметр жесткости, $r_{e}$ - равновесная длина связи. Также потенциалом Морзе представлялось взаимодействие между
Таблица 1. Параметры потенциала Морзе, описывающие различные взаимодействия

\begin{tabular}{c|r|c|c}
\hline Взаимодействие & $D_{e},(\mathrm{meV})$ & $\alpha, \mathrm{nm}^{-1}$ & $r_{e}, \mathrm{~nm}$ \\
\hline${ }^{*} \mathrm{Si}^{(1)}-\mathrm{Si}^{(2)}$ & 227.40 & 44.992 & 0.154 \\
$\mathrm{Si}-\mathrm{C}$ & 925.20 & 20.820 & 0.253 \\
$\mathrm{Li}-\mathrm{C}$ & 1258.51 & 17.074 & 0.206 \\
$\mathrm{Li}-\mathrm{Si}$ & 309.30 & 36.739 & 0.116
\end{tabular}

Примечание. Верхний индекс указывает на принадлежность к первому или второму листу силицена.

ионом $\mathrm{Li}^{+}$и атомами $\mathrm{Si}$ и С. Параметры этого потенциала определялись, исходя из соответствующих данных для описания взаимодействий $\mathrm{Li}-\mathrm{Li}[20], \mathrm{Si}-\mathrm{Si}[21]$ и $\mathrm{C}-\mathrm{C}$ [22]. Если известны параметры потенциала Морзе для представления взаимодействий между атомами сорта $A$ и между атомами сорта $B$, то параметры $A B$ взаимодействий получаются из простых интерполяционных соотношений [21]

$$
\begin{aligned}
D_{e}^{A B} & =\left(D_{e}^{A} D_{e}^{B}\right)^{1 / 2}, \\
\alpha^{A B} & =\frac{1}{2}\left(\alpha^{A}+\alpha^{B}\right), \\
r_{e}^{A B} & =\left(r_{e}^{A} r_{e}^{B}\right)^{1 / 2} .
\end{aligned}
$$

Используемые в настоящей работе параметры потенциала Морзе даны в табл. 1.

На основе данных сканирующей туннельной микроскопии было установлено, что после реконструкции различные участки монослоев $\mathrm{Si}$ на поверхностях $\mathrm{Ag}$ (1 111$)$ имеют разную структуру [23,24]. Среди них наибольшую площадь занимала структура, определенная как $(4 \times 4)$ силицен/Ag, которая соответствует силиценовой сверхячейке $(3 \times 3)$ на сверхячейке $\mathrm{Ag}(4 \times 4)$. Эта структура состоит из 18 атомов $\mathrm{Si}$, расположенных в двух подрешетках. Единичная ячейка такой структуры силицена имеющая форму ромба, содержит 18 атомов $\mathrm{Si}$. Шесть атомов $\mathrm{Si}$ единичной ячейки смещены на расстояние $0.064 \mathrm{~nm}$ перпендикулярно поверхности, а остальные атомы $\mathrm{Si}$ находятся на одной и той же (исходной) плоскости. Если в верхнем листе двухслойного силицена выступающие над исходной поверхностью атомы Si смещены вверх, то в нижнем листе такие атомы выдвигаются вниз. Мы провели ряд расчетов для совершенного силиценового канала, чтобы выбрать оптимальное расстояние между листами силицена $h_{g}$. Расчеты были начаты с зазора $0.60 \mathrm{~nm}$ и закончены значением $h_{g}=0.85 \mathrm{~nm}$ с шагом $\Delta h_{g}=0.05 \mathrm{~nm}$. Напряженность электрического поля составляла $10^{5} \mathrm{~V} / \mathrm{m}$. Ион начал полностью проходить канал при зазоре $h_{g}=0.70 \mathrm{~nm}$. Это расстояние значительно превышает дистанцию $0.2481 \mathrm{~nm}$ между листами двухслойного силицена с $A B$-укладкой, получаемую из расчетов в рамках теории функционала плотности [25]. В дальнейшем для исследования движения иона в дефектном силиценовом 
канале диапазон изменения зазора был сокращен до $0.70 \leq h_{g} \leq 0.85 \mathrm{~nm}$.

Листы совершенного силицена, содержащие по 300 атомов, имели прямоугольную форму $4.7 \times 4.0 \mathrm{~nm}$ (вдоль каждой кромки листа располагалось двенадцать атомов). Девять моно- или поливакансий располагались приблизительно равномерно по площади силиценового листа, так что число атомов $\mathrm{Si}$ в каждом листе составляло 291, 282, 273 и 246 для листов с моно-, би-, трии гексавакансиями соответственно. Постоянное электрическое поле напряженностью $10^{5} \mathrm{~V} / \mathrm{m}$ разгоняло ион $\mathrm{Li}^{+}$ вдоль оси $0 x$, ориентированной по направлению ,зигзаг“ силиценовых листов. Силиценовые листы располагались в соответствии с укладкой Бернала $(A B A B \ldots)$ точно таким же способом, как в объемном графите. Листы графена размещались параллельно листам силицена снаружи канала. Причем расстояние между листами силицена и графена было таким же как в работе [24], то есть $0.286 \mathrm{~nm}$. Фронтальные кромки листов графена и силицена совмещались. Каждый прямоугольный лист графена содержал 820 атомов, и вдоль каждой его кромки размещалось 20 атомов С. Первоначальное местоположение иона соответствовало высоте $h_{g} / 2$. При $t=0$ ион находился в точке с координатами $x=0.198 \mathrm{~nm}$, $y=2.245 \mathrm{~nm}$, то есть был слегка вдвинут в силиценовый канал приблизительно на середине ширины листа.

Стандартные коды программы LAMMPS позволяют делать расчеты до тех пор, пока все частицы в системе находятся в заданном замкнутом пространстве. Вычисления прекращаются, если по крайней мере одна из частиц покидает это пространство. Для того, чтобы этого не произошло, исследуемая функциональная модель силиценового электрода помещалась в прямоугольный контейнер. Более того, в начальный момент времени расстояние от любого атома $\mathrm{Si}$ или $\mathrm{C}$, принадлежащего границе силицен-графитовой системы, до ближайшей стенки контейнера составляло не менее $0.5 \mathrm{~nm}$. Расстояние от атомов $\mathrm{Si}$ верхнего листа силицена до верхней стенки контейнера составляло $1.15 \mathrm{~nm}$. Взаимодействие иона $\mathrm{Li}^{+}$со стенкой происходило только тогда, когда он приближался к стенке на расстояние $0.25 \mathrm{~nm}$. Ион лития мог взаимодействовать со стенкой контейнера в соответствии с потенциалом Леннарда-Джонса (12-6) с параметрами $\sigma=0.1 \mathrm{~nm}, \varepsilon=1 \mathrm{eV}$. Эти параметры не имеют отношения к какому-либо реальному материалу и выбраны эмпирически для получения адекватной модели, воспроизводящей движение иона по силиленовому каналу. Если расстояние до стенки было меньше $0.1 \mathrm{~nm}$, сила отталкивания воздействовала на атом (ион), и в конечном итоге направление его движения изменялось на противоположное. Численное решение уравнений движения выполнялось методом Рунге-Кутты четвертого порядка с временным шагом $\Delta t=1 \cdot 10^{-16} \mathrm{~s}$. Длительность каждого расчета с величиной зазора $h_{g}$ составляла 1 млн. временных шагов. Силиценовый канал, имеющий зазор $0.70 \leq h_{g} \leq 0.85 \mathrm{~nm}$, располагался на графитовой подложке.
Для расчета напряжений, возникающих в силицене, листы разбивались на элементарные площадки. Напряжения $\sigma_{n \alpha}(l)$, возникающие под действием сил в направлении $\alpha$ ( $\alpha$ принимает значения $x, y, z)$, вычисляются на каждой площадке с номером $l$, имеющей ориентацию $n$. В этих расчетах используются произведения проекций скоростей атомов и проекций сил $f_{i j}^{\alpha}$, действующих на площадку $l$ со стороны других атомов при соблюдении соответствующих условий [26]

$$
\sigma_{n \alpha}(l)=\left\langle\sum_{i}^{k} \frac{1}{\Omega}\left(m v_{n}^{i} v_{\alpha}^{i}\right)\right\rangle+\frac{1}{S_{l}}\left\langle\sum_{i}^{k} \sum_{j \neq i}^{\left(u_{i} \leq u, j \geq u\right)}\left(f_{i j}^{\alpha}\right)\right\rangle .
$$

Здесь $k$ - количество атомов на площадке с номером $l$, $\Omega$ - объем, приходящийся на атом, $m$ - масса атома, $v_{\alpha}^{i}-\alpha$-проекция скорости $i$ го атома, $S_{l}-$ площадь площадки $l$. Условия суммирования по $j$ в последней сумме выражения (2) отражены как в нижнем, так и в верхнем индексе суммы; сила, возникающая при взаимодействии атомов $i$ и $j$, проходит через $l$-ю площадку; $u_{i}$ - текущая координата атома $i$, в верхнем индексе суммы $u$ обозначает координату пересечения прямой, проходящей через центры атомов $i$ и $j$, с площадкой $l$.

Структурный анализ малых объектов может быть выполнен с использованием метода статистической геометрии, основанного на построении многогранников Вороного (МВ) [27]. В случае многоатомной системы атомы одного типа могут играть роль центров многогранников, а атомы другого типа используются для определения граней многогранников. Например, в случае системы „Lі-силиценовый канал“ целесообразно использовать атом лития в качестве центра и выбрать ближайших соседей среди атомов $\mathrm{Si}$. Такие гибридные многогранники практически всегда можно построить, так как атомы $\mathrm{Si}$ находятся по обеим сторонам от атома $\mathrm{Li}$, а листы силицена не представляют собой идеальную плоскость. Однако в этом случае гибридные многогранники не являются многогранниками Вороного, поскольку они полностью не заполняют все пространство, занимаемое молекулами, без пустот и перекрытий. Грани МВ определяют циклические структуры, образованные из атомов того же сорта, к которому принадлежит центральный атом (центр МВ), в то время как поверхности рассматриваемых гибридных полиэдров с центром, совпадающим с положением атома Li определяют кольца, состоящие из атомов Si. Многогранники Вороного можно построить и в многокомпонентной системе, если размеры всех атомов одинаковы. Мы не будем учитывать разницу в размерах атомов $\mathrm{Li}$ и $\mathrm{Si}$ при построении многогранников, т. е. будем рассматривать MB.

Метод построения многогранников Вороного можно использовать не только для изучения структуры ближнего порядка „бесконечных“ или конечных объектов, но также для того, чтобы проследить за ближним порядком в рельефе канала при движении по нему иона лития. Для этого нужно совместить центр конструируемого 
многогранника с ионом $\mathrm{Li}$, а в качестве геометрических соседей для иона выбирать атомы $\mathrm{Si}$ канала, по которому движется ион. Многогранники строятся через равные промежутки времени до тех пор, пока ион не покинет канал. В итоге, движение иона по каналу под действием постоянного электрического поля может быть представлено в форме распределений элементов многогранников Вороного. Сопоставление таких распределений, полученных для разных каналов, даст представление о причинах производимого каналом тормозного эффекта. Поэтому будем называть такое исследование статистико-геометрическим методом представления тормозного эффекта в канале.

\section{3. Результаты расчета}

Общий вид системы „спаренные листы силицена с бивакансиями на графите“ после 32.5 ps от начала ввода в канал с первоначальным зазором $h_{g}=0.80 \mathrm{~nm}$ иона $\mathrm{Li}^{+}$показан на рис. 1. Ион $\mathrm{Li}^{+}$, входящий в канал с левой стороны (см. рис. 1), совершает не только горизонтальные, но и вертикальные перемещения, двигаясь в электрическом поле, вектор напряженности которого направлен вдоль оси $0 x$. Видно, что в ходе расчета происходят заметные вертикальные смещения атомов $\mathrm{Si}$ как в верхнем, так и в нижнем листе силицена. Причем при переходе от силицена с моновакансиями к силицену с гексавакансиями величина смещений увеличивается. Аналогичная картина наблюдается для систем с подложками $\mathrm{Ag}$ (001) и $\mathrm{Ag}$ (111) [15]. При наличии как серебряной, так и графитовой подложки происходят перестройки атомов $\mathrm{Si}$ в плоскости нижнего

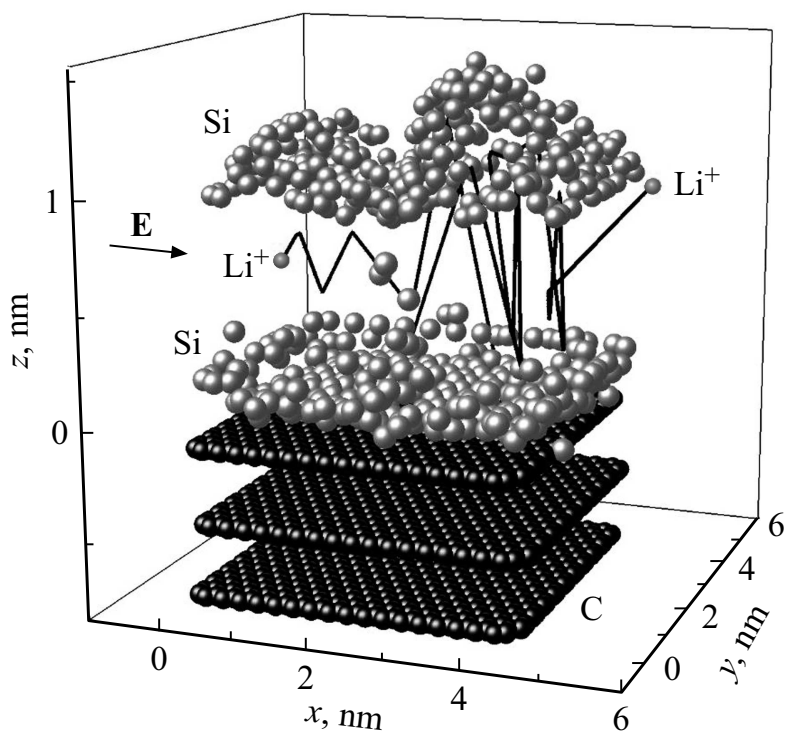

Рис. 1. Конфигурация системы „спаренные листы силицена с бивакансиями на графите“ при первоначальном зазоре между листами $h_{g}=0.80 \mathrm{~nm}$ изображена вместе с траекторией движения иона лития по каналу за время 32.5 ps. Постоянное электрическое поле действует вдоль оси $0 x$.

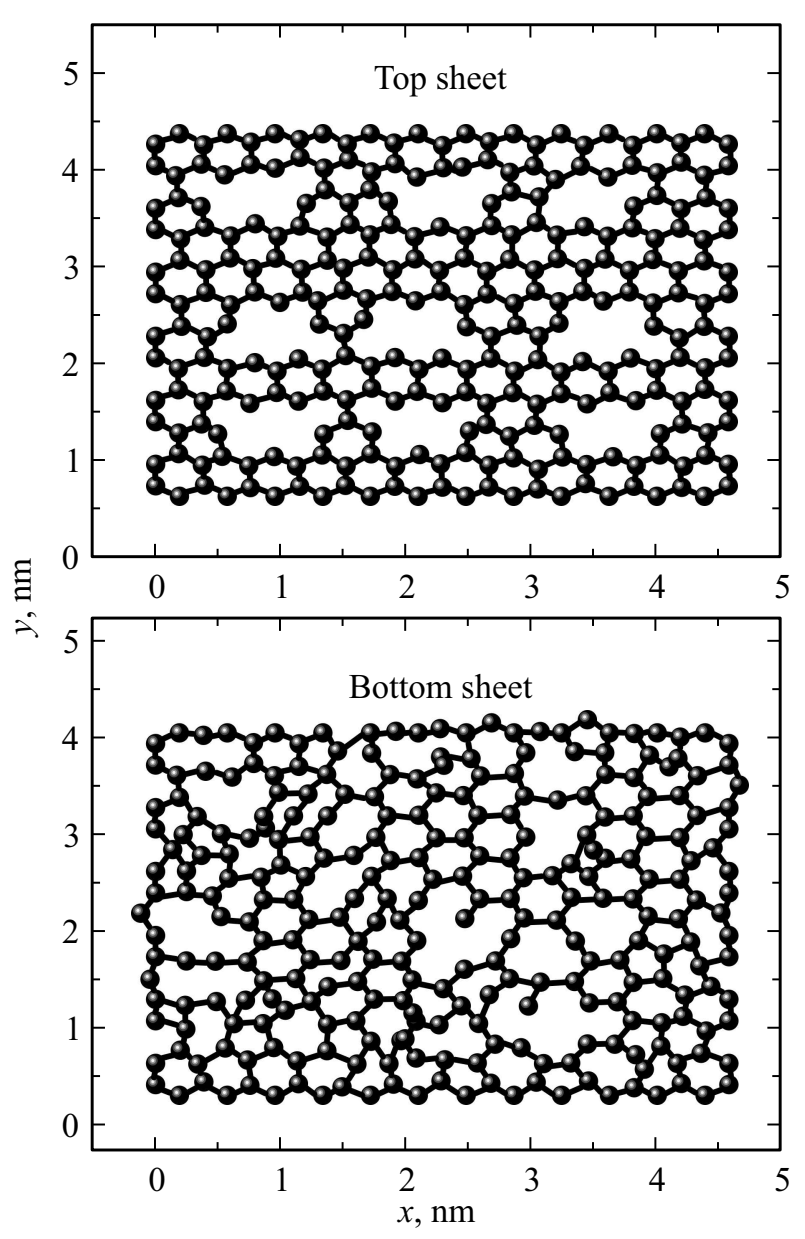

Рис. 2. Проекции на плоскость $x y$ верхнего и нижнего листов силицена с тривакансиями на графитовой подложке с межслойной щелью $0.75 \mathrm{~nm}$ в момент времени $100 \mathrm{ps}$.

листа силицена. На серебряных подложках при наличии дефектов в верхнем листе силицена также наблюдаются значительные структурные перестройки.

На рис. 2 представлены проекции на плоскость $x y$ верхнего и нижнего листов силицена с тривакансиями при $h_{g}=0.80 \mathrm{~nm}$, полученные к моменту времени 100 ps. Силицен помещался на графитовую подложку. Видно, что подложка оказывает сильное влияние на структуру нижнего листа силицена, моментальный снимок которого к концу расчета сильно изменился. В то же время верхний лист сохранил не только все девять дефектов, но также в значительной степени их размер и форму. Число пор в лежащем на подложке нижнем листе силицена увеличилось, но размер большинства пор стал меньше, чем размер исходной тривакансии. Большая часть образовавшихся в нижнем силицене пор имеет извилистые границы, но также существуют поры с гладкими границами. Примечательно, что в сильно перестроенной структуре нижнего листа появились атомы с двумя оборванными связями. Таким образом, несовместимость периодов решеток силицена и графена (поверхности графита) оказывает сильное влияние 

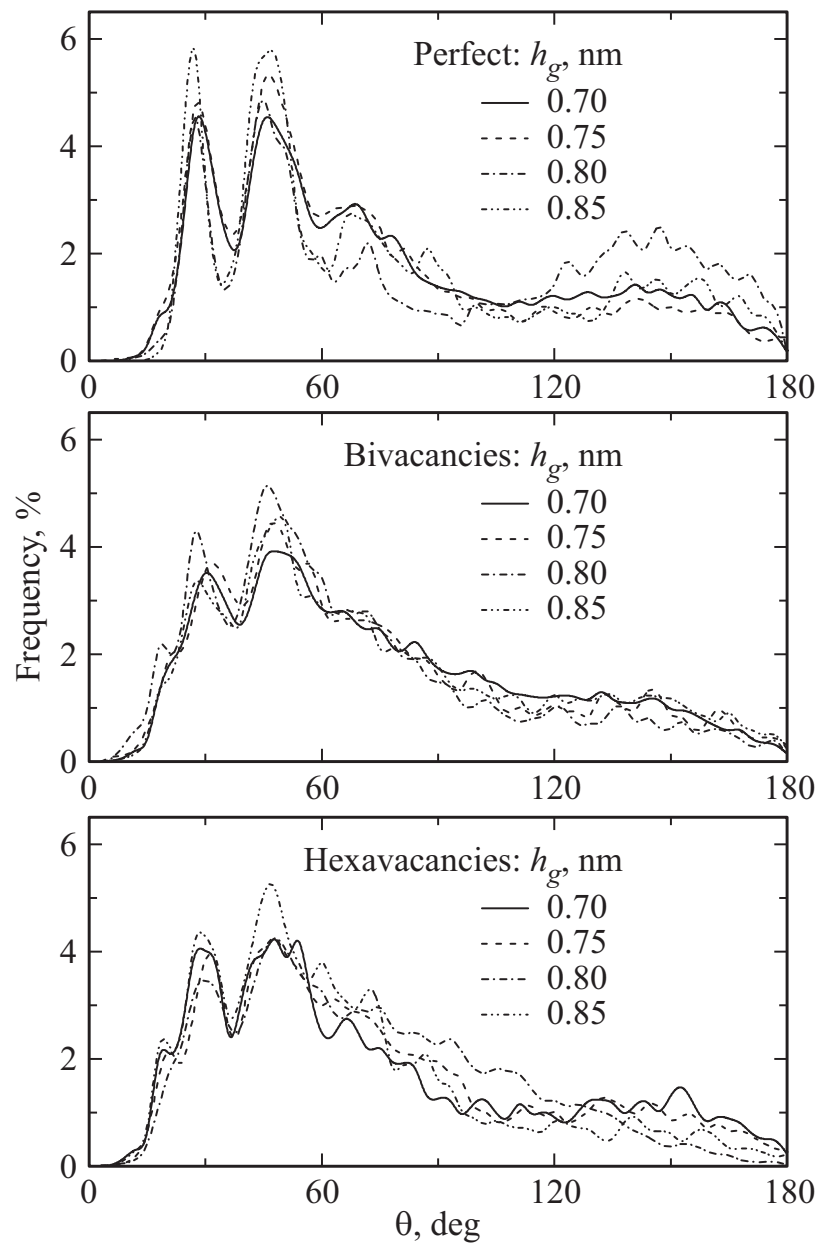

Рис. 3. Угловые распределения геометрических соседей иона лития при его движении по различным силиценовым каналам.

на структуру дефектного силицена после прохождения ионом лития всего канала.

Угловые распределения геометрических соседей, встречаемых на пути иона в каналах с совершенными силиценовыми стенками, а также с листами силицена, содержащими би- и гексавакансии, показаны на рис. 3. Рассматриваются углы, в вершинах которых находится ион, а стороны связывают его с ближайшими геометрическими соседями. Из рисунка видно, что эти характеристики значительно изменяются не только при изменении дефектности стенок канала, но также при изменении его зазора. Как правило, интенсивность пиков, лежащих в окрестности углов 30 и $45^{\circ}$, усиливается с увеличением величины зазора. Однако рост этих пиков замедляется с увеличением размера дефектов. Эти пики формируются благодаря наиболее дальним геометрическим соседям. Число таких соседей увеличивается при расширении канала, и уменьшается из-за увеличения размера дефектов. Поведение рассматриваемых пиков в основном обусловлено именно этими факторами. В диапазоне углов $120^{\circ} \leq \theta \leq 180^{\circ}$ угловые спектры проявляют большую стабильность при увеличении зазора канала, так как расширение канала слабо сказывается на количестве наиболее близких геометрических соседей иона.

На рис. 4 показаны распределения МВ, сформированных вокруг движущегося по каналу иона $\mathrm{Li}^{+}$, по числу граней. Эти распределения также характеризуют число атомов $\mathrm{Si}$, попадающих в ближайшее окружение иона $\mathrm{Li}^{+}$. В случае канала со стенками из совершенного силицена ион $\mathrm{Li}+$ наиболее часто оказывается окружен девятью атомами $\mathrm{Si}$, если зазор $h_{g} \leq 0.75 \mathrm{~nm}$. Однако при увеличении зазора определенность в установлении наиболее высоких значений числа окружающих ион атомов $\mathrm{Si}$ снижается. Так при $h_{g}=0.80 \mathrm{~nm}$ окружение иона $\mathrm{Li}^{+}$13-ю и 15-ю атомами $\mathrm{Si}$ встречается наиболее часто, а при $h_{g}=0.85 \mathrm{~nm}$ доминирует окружение $\mathrm{Li}^{+}$четырьмя атомами Si. Появление вакансионных дефектов в стенках канала делает $\mathrm{Si}$-окружение иона еще более непредсказуемым. Только в канале, образованном листами силицена с моновакансиями, с $h_{g}=0.70 \mathrm{~nm}$ наиболее вероятно окружение иона пятнадцатью атомами $\mathrm{Si}$. В остальных случаях, как правило, существует большая неопределенность в установлении этой характеристики.

Исключение мелких геометрических элементов в многогранниках Вороного позволяет перейти к анализу более вероятных геометрических соседей, обнаруживаемых в окружении иона $\mathrm{Li}^{+}$. Усеченные многогранники, в которых отсутствуют мелкие грани, имеют более компактные $n$-распределения с максимальным значением $n \leq 25$ (рис. 5). Однако и в этом случае трудно найти закономерность в зависимостях числа наиболее вероятных граней от величины зазора $h_{g}$ силиценового канала и от размера дефектов в листах силицена. Из рис. 5 видно, что пики с интенсивностью, большей $15 \%$, существуют только в $n$-распределениях для канала с совершенными стенками и со стенками, имеющими моновакансии.

Распределение колец, образованных атомами $\mathrm{Si}$ вокруг иона $\mathrm{Li}^{+}$, движущегося по каналу со стенками из совершенного и дефектного силицена при различных зазорах $h_{g}$ (m-распределение) показано на рис. 6 . Для зазора $h_{g}=0.70 \mathrm{~nm}$ наблюдается смещение пика $m$-распределения от значения $m=10$ до значений $m=5$ и 4 при переходе от канала с совершенными силиценовыми стенками к каналу со стенками, содержащими бивакансии и гексавакансии соответственно. В остальных показанных на рис. 6 случаях, максимум $m$-распределения лежит в диапазоне значений $3 \leq m \leq 6$. Влияние би- и гексавакансий на формирование ближнего порядка вокруг движущегося по каналу иона $\mathrm{Li}^{+}$ оказывается наиболее ощутимым в самом узком из рассматриваемых здесь каналов (при $h_{g}=0.70 \mathrm{~nm}$ ).

Статистика $m$-членных колец, существующих вокруг движущегося иона $\mathrm{Li}^{+}$значительно проясняется в случаe перехода от многогранников Вороного к усеченным многогранникам. На рис. 7 представлены распределения для граней усеченных многогранников $\left(m_{t r}\right.$-распределения), характеризующих движение иона $\mathrm{Li}^{+}$в силиценовых каналах с различными зазорами $h_{g}$, стенки которых образованы листами совершенного и дефектного 

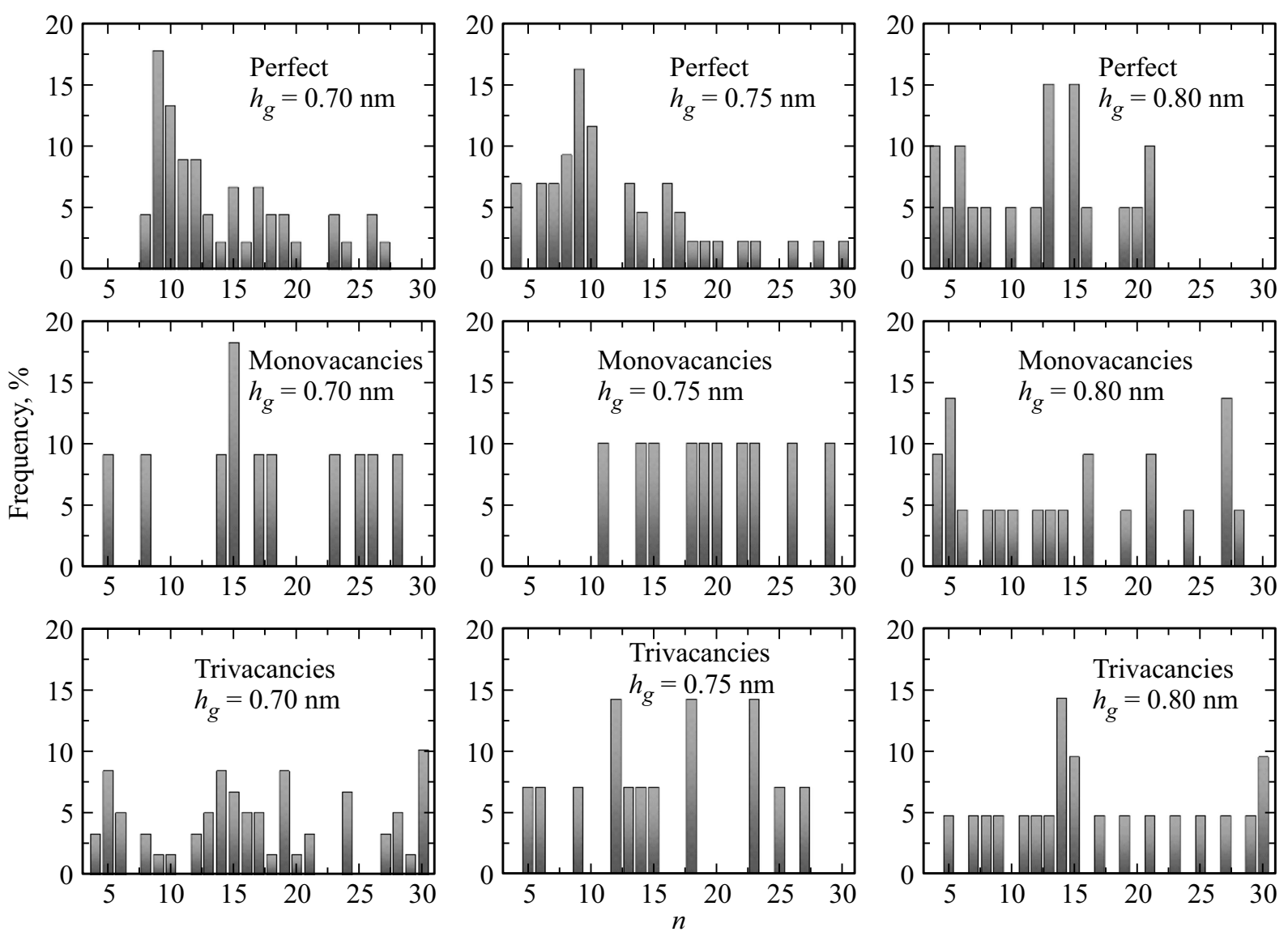

Рис. 4. Частота появления числа $n$ атомов $\mathrm{Si}$, включаемых в ближайшие геометрические соседи иона $\mathrm{Li}^{+}$при его движении по совершенному силиценовому каналу и каналу, стенки которого содержат различные вакансионные дефекты. $h_{g}$ - ширина зазора силиценового канала.

силицена. В этом случае четырехзвенные кольца преобладают в самом узком из рассматриваемых каналов $\left(\right.$ c $\left.h_{g}=0.70 \mathrm{~nm}\right)$ вне зависимости от того, содержат ли его стенки дефекты. Четырех- и пятизвенные кольца являются наиболее частыми в остальных, показанных на рис. 7, случаях. Причем, расширение канала чаще приводит к появлению пика $m_{t r}$-распределения при $m_{t r}=5$.

Распределения расстояний ( $r$-распределения) до ближайших геометрических соседей иона $\mathrm{Li}^{+}$при различных зазорах и типах каналов показаны на рис. 8. Появление дефектов в стенках канала приводит к уменьшению стандартного отклонения $r$-распределения. В среднем сокращение стандартного отклонения в каналах из дефектного силицена по отношению к каналу из совершенного силицена составило 19\%. Изменение зазора канала приводит к неоднозначному изменению расстояния $r_{m p}$ от иона до наиболее вероятного геометрического соседа. Усредненное по зазору канала значение $r_{m p}$ составляет $0.588,0.542$ и $0.546 \mathrm{~nm}$ для совершенного силиценового канала и каналов с моно- и гексавакансиями соответственно.

Распределение наиболее значимых напряжений $\sigma_{z z}(x)$, действующих в плоскости листов силицена при движении иона $\mathrm{Li}^{+}$по каналу, показано на рис. 9. Элементар- ные площадки вытянуты вдоль оси 0у. Электрическое поле напряженностью $10^{5} \mathrm{~V} / \mathrm{m}$ действует вдоль оси $0 x$. Здесь не прослеживается явная зависимость интенсивности флуктуаций напряжения $\sigma_{z z}$ от величины канального зазора и от типа дефектов, присутствующих в силицене. В силу того, что силы, создающие напряжение $\sigma_{z z}$, как правило, направлены вниз из-за притяжения силицена к графитовой подложке, значения напряжения $\sigma_{z z}$ почти всегда отрицательны. Наиболее сильные локальные напряжения, появляющиеся при движении иона $\mathrm{Li}^{+}$по каналу, по абсолютной величине составляют не более $1.65 \mathrm{GPa}$ или $0.693 \mathrm{~N} / \mathrm{m}$. Эта величина в 8.4 раза ниже, чем предел прочности силицена при растяжении, полученный в работе [28]. Таким образом, при движении иона $\mathrm{Li}^{+}$по каналу, образованному листами совершенного или дефектного силицена, не появляется опасных локальных напряжений, способных вызвать разрушение силицена.

\section{4. Обсуждение}

Движение иона $\mathrm{Li}^{+}$в плоском силиценовом канале происходит много труднее, чем в аналогичном графе- 

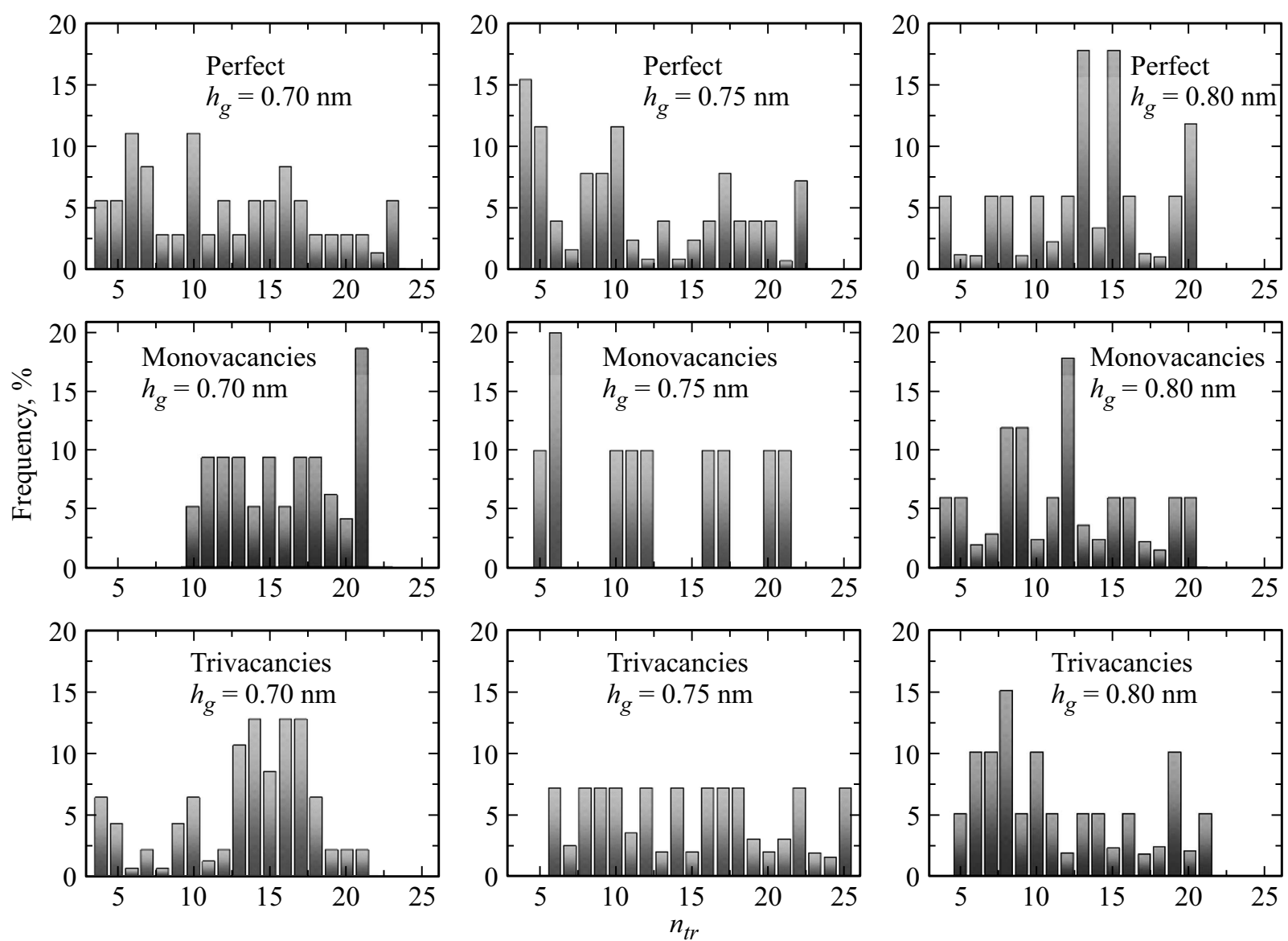

Рис. 5. Частота появления числа $n$ атомов $\mathrm{Si}$, включаемых в более вероятные геометрические соседи иона $\mathrm{Li}^{+}$при его движении по совершенному силиценовому каналу и каналу, стенки которого содержат различные вакансионные дефекты. $h_{g}-$ ширина зазора силиценового канала.

новом канале. Так для прохождения ионом $\mathrm{Li}^{+}$совершенного графенового канала, не имеющего подложки, длиной $\sim 4 \mathrm{~nm}$ достаточна напряженность электрического поля $E=10^{3} \mathrm{~V} / \mathrm{m}$ при зазоре между листами $h_{g}=0.60 \mathrm{~nm}$ [29]. Ион лития полностью проходит находящийся в вакууме совершенный силиценовый канал аналогичной длины при величине $E=10^{5} \mathrm{~V} / \mathrm{m}$, начиная с зазора между стенками канала $h_{g}=0.75 \mathrm{~nm}$ [30]. Движение иона $\mathrm{Li}^{+}$по силиценовому каналу еще более усложняется в случае присутствия дефектов вакансионного типа в стенках канала. Так при зазоре $h_{g}=0.80 \mathrm{~nm}$ и напряженности поля $E=10^{5} \mathrm{~V} / \mathrm{m}$ ион проходит канал за время меньшее $35 \mathrm{ps}$, если его стенки содержат монои бивакансии, но не может покинуть канал при наличии в его стенках три- и гексавакансий даже за 100 ps [31]. В случае, когда дефектный силицен с обоих сторон поддерживался однослойным совершенным графеном, ион лития полностью проходил (за время $<65$ ps) более узкий канал $\left(h_{g}=0.75 \mathrm{~nm}\right)$ со стенками, включающими моно- и бивакансии. Однако такой канал со стенками, имеющими три- и гексавакансии преодолеть за $100 \mathrm{ps}$ ион $\mathrm{Li}^{+}$не мог [32]. Таким образом, применение силицена вместо графена в качестве анодного материала требует расширения зазора между смежными листами и значительного увеличения напряженности электрического поля.

В настоящем исследовании мы установили, что при напряженности электрического поля $10^{5} \mathrm{~V} / \mathrm{m}$ еще более узкий силиценовый канал с зазором $h_{g}=0.70 \mathrm{~nm}$ обеспечивает прохождение иона лития, если канал поддерживается графитовой подложкой. В этом случае проходимость канала сохраняется не только в присутствии

Таблица 2. Время прохождения иона лития через силиценовый канал, находящийся на подложке из графита, ps

\begin{tabular}{l|c|c|c|c}
\hline \multirow{2}{*}{$\begin{array}{c}\text { Тип вакансий } \\
\text { в силицене }\end{array}$} & \multicolumn{4}{|c}{ Величина зазора, $\mathrm{nm}$} \\
\cline { 2 - 5 } & 0.7 & 0.75 & 0.8 & 0.85 \\
\hline Совершенный & 45.4 & 42.2 & 20.4 & 73.6 \\
Моновакансии & 10.3 & 12.2 & 24.3 & 16.5 \\
Бивакансии & 74.5 & не выходит & 35.2 & 16.8 \\
& & из канала & & \\
Тривакансии & 64.0 & 13.8 & 21.2 & 25.9 \\
Гексавакансии & 16.7 & 18.2 & не выходит & 19.4 \\
& & & из канала &
\end{tabular}



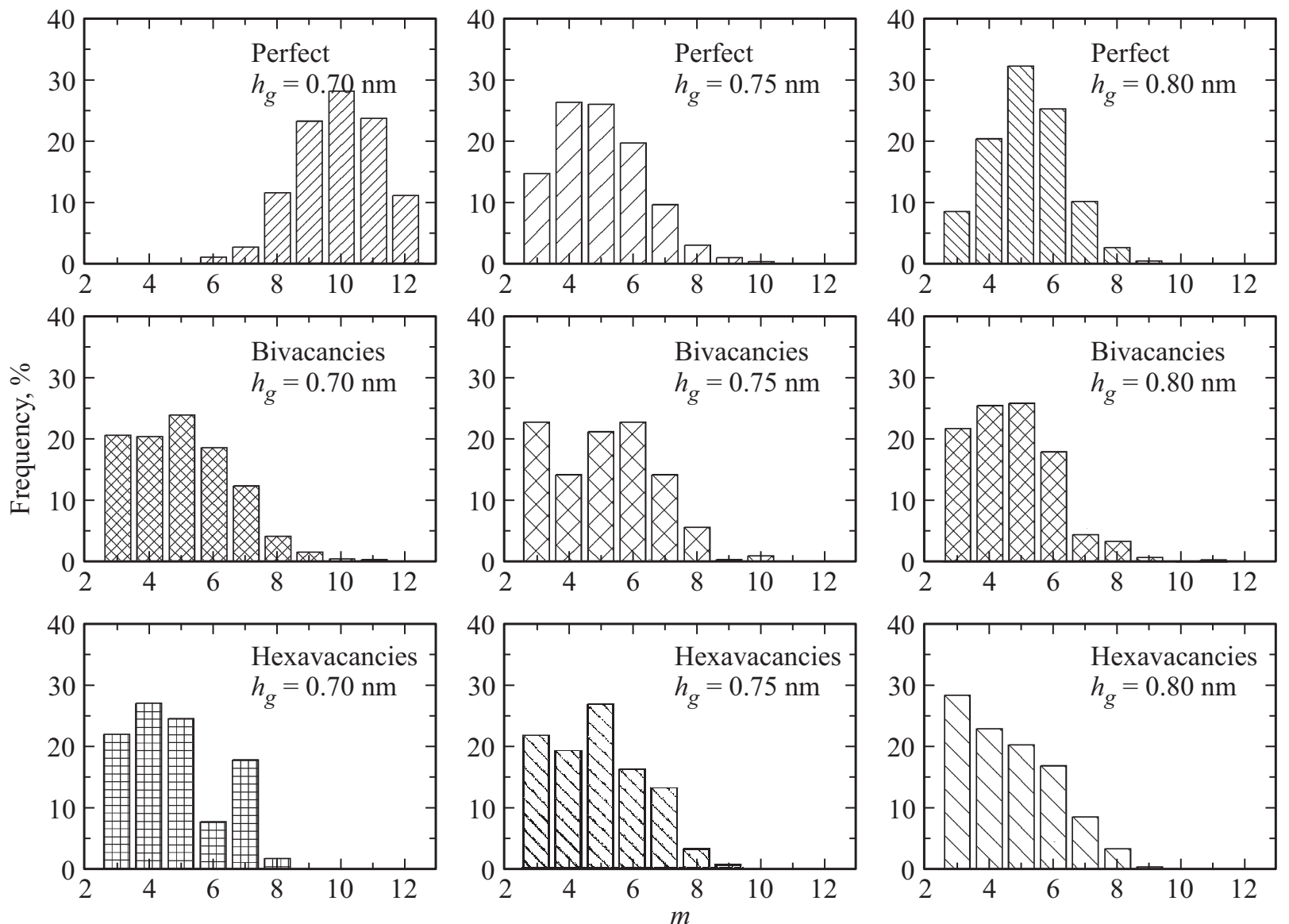

Рис. 6. Число атомов $\mathrm{Si}$, формирующих кольца вокруг иона $\mathrm{Li}^{+}$в каналах с различными зазорами, стенки которых содержат различные дефекты.

моно- и бивакансий в его стенках, но также при наличии в них три- и гексавакансий. Времена прохождения ионом лития силиценового канала, находящегося на графитовой подложке, представлены в табл. 2. Наибольшее время прохождения канала с зазором $0.70 \mathrm{~nm}(74.5 \mathrm{ps})$ зафиксировано, когда в его стенках содержатся бивакансии. Ион $\mathrm{Li}^{+}$остается в силиценовом канале с зазором $h_{g}=0.75 \mathrm{~nm}$ и стенками, содержащими бивакансии, в течение $100 \mathrm{ps}$, но достаточно быстро проходит этот канал (быстрее $36 \mathrm{ps}$ ) в присутствии три- и гексавакансий в его стенках. Тривакансии в стенках канала не мешают иону $\mathrm{Li}^{+}$полностью проходить его при всех рассматриваемых зазорах $(0.70-0.85 \mathrm{~nm})$ в течение времени, не превышающего 64 ps. В случае гексавакансий непроходимость канала за 100 ps появляется только при зазоре $h_{g}=0.80 \mathrm{~nm}$. Сильное взаимодействие с подложкой разрушает нижний и верхний листы как совершенного, так и дефектного силицена на подложках $\operatorname{Ag}(001)$ и $\operatorname{Ag}(111)$ и делает этот материал непригодным для конструирования анода литий-ионной батареи [15]. Таким образом, использование графитовой подложки для поддержки силиценового канала создает лучшую проходимость канала ионом лития, чем применение серебряной подложки или двухсторонней поддержки силиценового канала листами графена.

Во всех рассмотренных случаях движение иона $\mathrm{Li}^{+}$ по силиценовому каналу нельзя рассматривать как движение частицы в разупорядоченной среде. На это указывает появление двух пиков с местоположениями $<50^{\circ}$ в угловом распределении ближайших геометрических соседей. Для разупорядоченных систем существует только один пик в этой области [33]. Рассчитанные распределения многогранников по числу граней распространяются на широкую область значений, в отдельных случаях достигая значения $n=30$. Такое поведение $n$-распределения также не характерно для разупорядоченных систем. Кроме того, многие из рассчитанных $n$-распределений имеют большое количество равновероятно распределенных типов граней. Последняя черта скорее всего является спецификой 2D-систем, которая сохраняется и при переходе к рассмотрению статистических распределений для усеченных многогранников. Вид распределения граней МВ по числу сторон в значительной мере определяется как величиной зазора $h_{g}$, так и типом дефектов, содержащихся в стенках канала. Обе эти характеристики также оказывают влияние на вид $m$-распределений, построенных для усеченных 

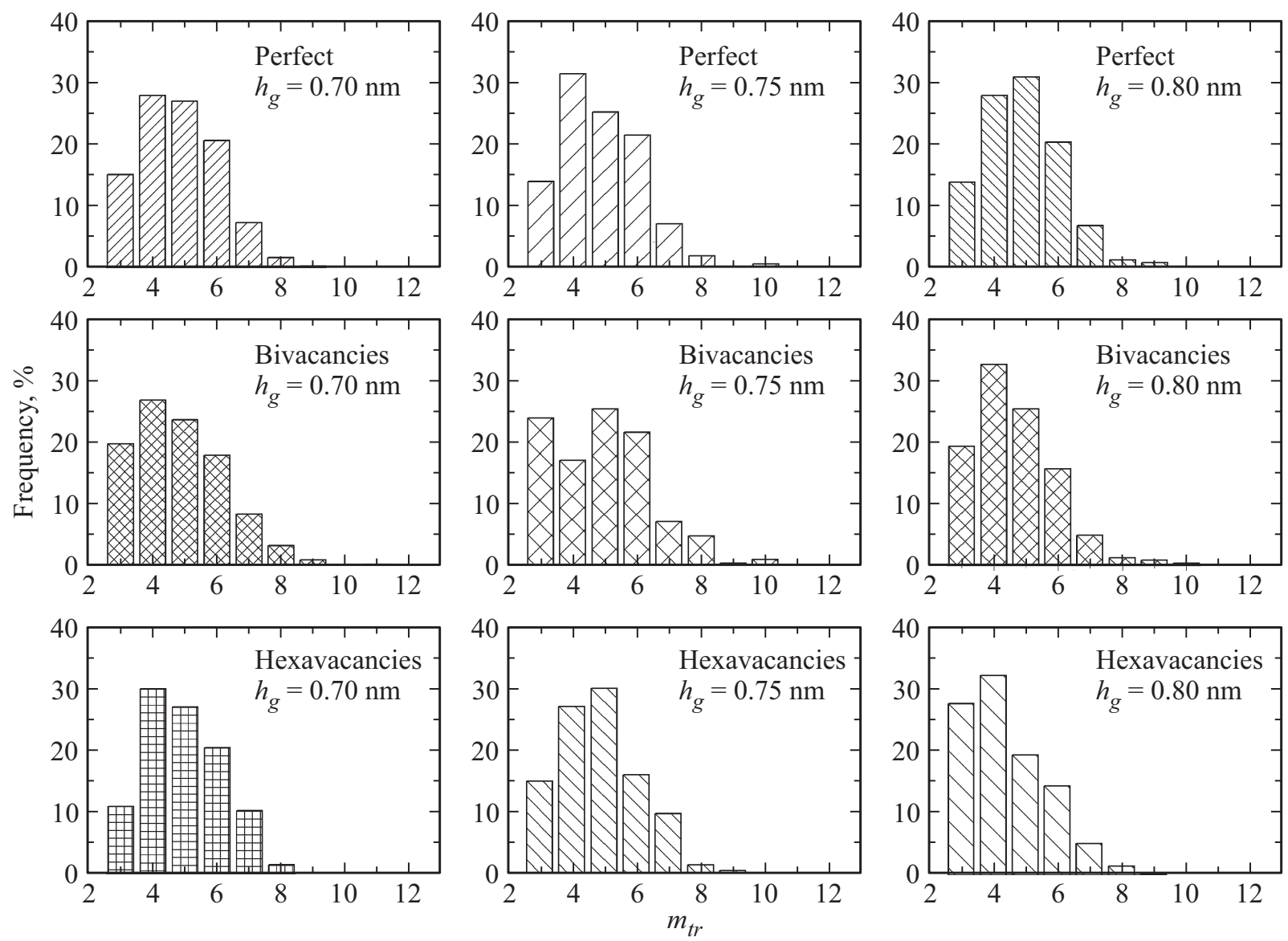

Рис. 7. Число атомов $\mathrm{Si}$, образующих осредненные кольца вокруг иона $\mathrm{Li}^{+}$в каналах с различными зазорами, стенки которых содержат различные дефекты.

многогранников. Аналогичное утверждение в целом справедливо для распределений расстояний от иона до ближайших геометрических соседей - атомов $\mathrm{Si}$.

Расположение силиценового канала на графите существенно снижает максимальные локальные напряжения , появляющиеся в стенках канала при движении по нему иона $\mathrm{Li}^{+}$. Так полученная при размещении силиценового канала на графите средняя величина $\left|\sigma_{z z}\right|_{\max }$ меньше аналогичной характеристики, обнаруженной в случае канала на подложках $\mathrm{Ag}(001)$ и $\mathrm{Ag}(111)$ приблизительно в 2.4 раза и в 5.3 раза уступает значению $\left|\sigma_{z z}\right|_{\max }$, установленному при использовании для поддержки канала графена $[15,32]$. Ослабление напряжения $\left|\sigma_{z z}\right|_{\max }$ при переходе к неметаллической подложке связано с существенно более слабыми взаимодействиями $\mathrm{Si}-\mathrm{C}$ по сравнению с взаимодействиями $\mathrm{Si}-\mathrm{Ag}$. Резкое снижение величины $\left|\sigma_{z z}\right|_{\max }$ в случае силиценового канала на графите можно объяснить удалением его второй стенки от подложки на расстояние, превышающее расстояние между силиценом и поддерживающим его графеном как минимум в 4.4 раза. Кроме того, начальное расстояние между нижним слоем силицена и графитом $r_{\mathrm{Si}-\mathrm{C}}=0.286 \mathrm{~nm}$ было больше, чем расстояние между силиценом и графеном в гибридном канале $(0.222 \mathrm{~nm})$. В связи с этим между атомами $\mathrm{Si}$ и $\mathrm{C}$ в силицено-графеновом канале моделировались ковалентное взаимодействие с использованием потенциала Терсоффа, тогда как в настоящей работе рассматривается вандерааальсово взаимодействие между атомами $\mathrm{Si}$ и С. Вследствие очень слабого влияния графитовой подложки на верхний лист силицена, структура этого листа может сохраняться в течение всего моделирования даже при наличии в листе крупных дефектов, таких как три- и гексавакансии.

\section{5. Заключение}

Предложен метод изучения структуры 2D материала по перемещению вдоль его плоскостей пробной частицы, роль которой в данном случае выполняет ион лития. Метод основан на построении многогранников Вороного, центры которых совпадают с местоположениями иона $\mathrm{Li}^{+}$. Сканирование листов совершенного силицена и силицена, содержащего дефекты вакансионного типа, с помощью перемещения иона лития в канале показало, что образованный листами канал имеет специфическую структуру, не похожую на структуру 3D упорядоченных или разупорядоченных материалов. Структура слоистых 2D-материалов характеризуется присутствием двух чет- 
ких пиков в угловом распределении ближайших геометрических соседей. Местоположения этих пиков ограничены условием $\theta_{a}, \theta_{2}<50^{\circ}$. Структура 2D-кремния описывается очень широким набором ближайших геометрических соседей, определяемых для движущегося центра. Вне зависимости от присутствия дефектов того или иного типа в листах силицена число геометрических соседей для иона лития лежит в диапазоне $4 \leq n \leq 30$. Для $n$-спектров 2D-кремния характерно заметное присутствие равновероятного распределения различных геометрических соседей. В зависимости от ширины зазора в канале и типа дефектов, содержащихся в его стенках, образующиеся вокруг иона наиболее вероятные кольца из атомов $\mathrm{Si}$ могут иметь от трех до десяти звеньев. Устранение мелкомасштабных флуктуаций, сопровождающих движение иона, сокращает диапазон изменения числа звеньев в наиболее часто встречаемых кольцах от четырех до пяти. Причем четырехзвенные кольца из геометрических соседей встречаются чаще, чем пятизвенные. Наиболее вероятные расстояния от иона до ближайших геометрических соседей могут заметно изменяться в зависимости от величины зазора в канале и типа дефектов, содержащихся в его стенках. Напряжения $\sigma_{z z}$, действующие в плоскости листов силицена и вызванные силами, направленными перпендикулярно этой плоско-

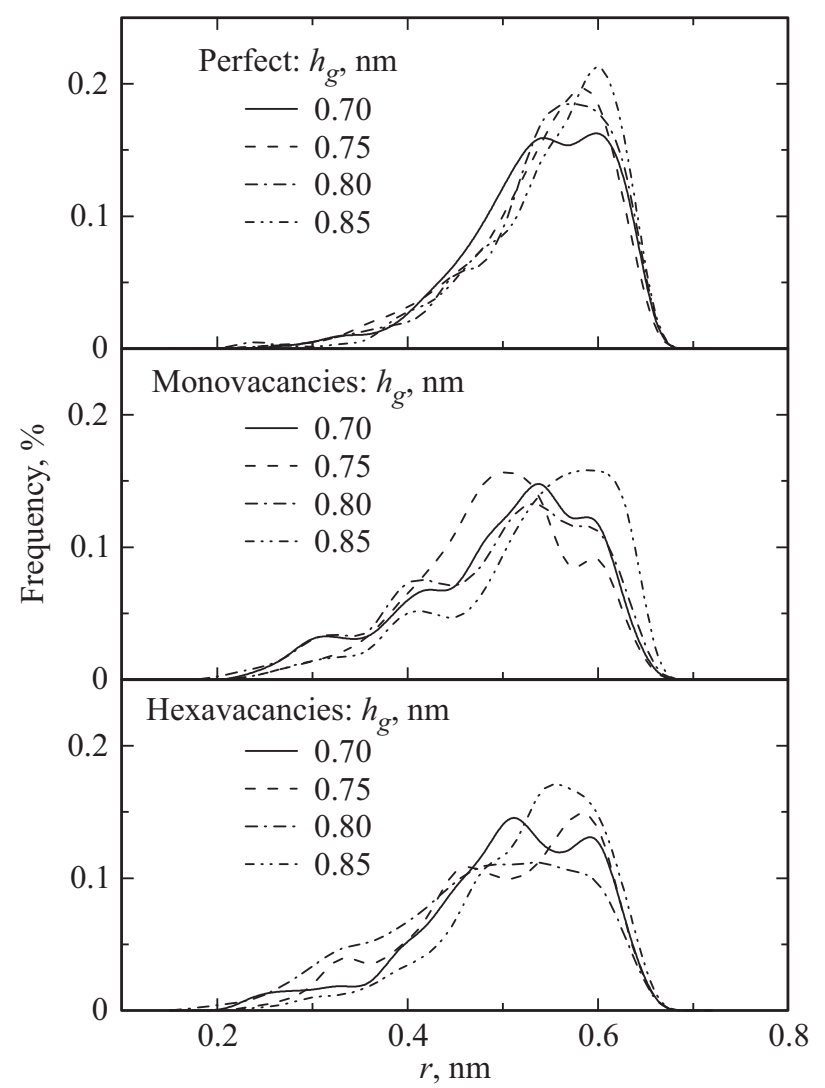

Рис. 8. Распределение расстояний до ближайших геометрических соседей иона $\mathrm{Li}^{+}$в канале со стенками из совершенного и дефектного силицена при различных зазорах $h_{g}$.

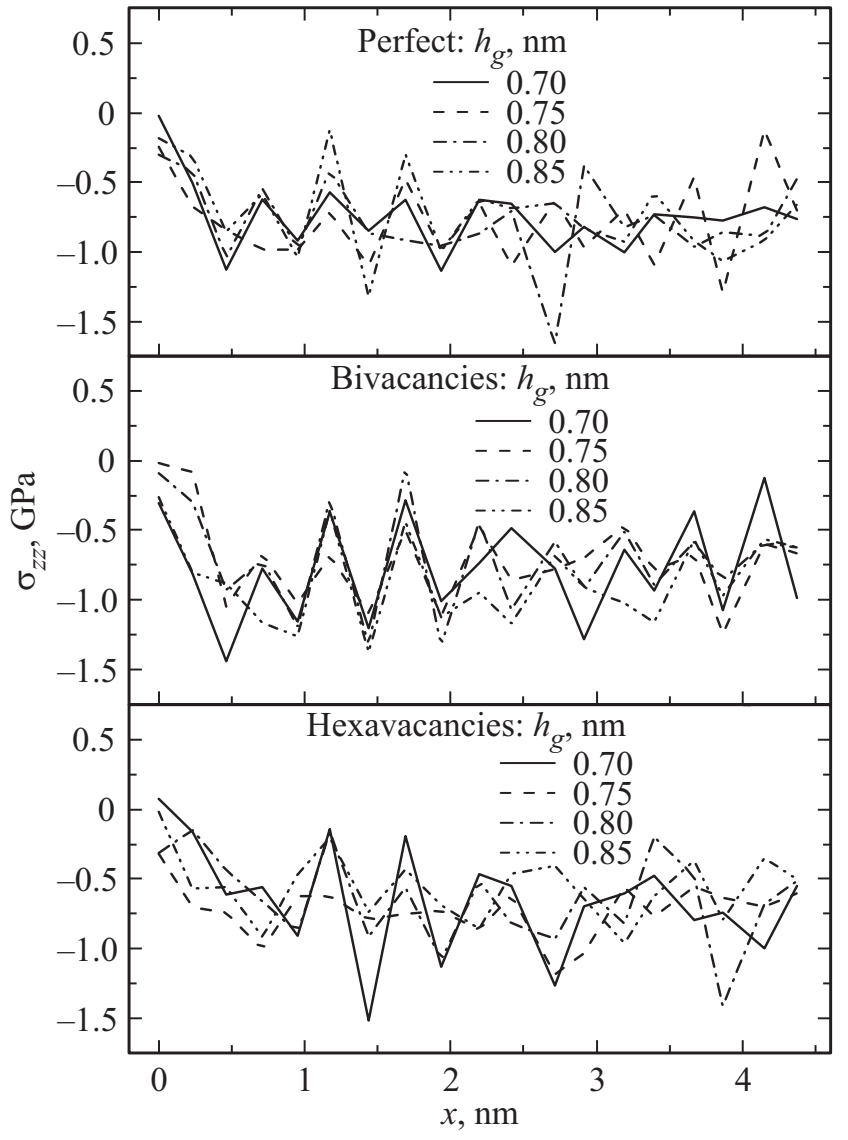

Рис. 9. Распределение напряжения $\sigma_{z z}(x)$, усредненного по обеим листам совершенного и дефектного силицена, вдоль оси $0 x$ (зигзагообразное направление), совпадающей с направлением напряженности электрического поля, при различных зазорах.

сти, являются наиболее значимыми при движении иона $\mathrm{Li}^{+}$вдоль силиценового канала. Локальные всплески напряжения $\sigma_{z z}$ заметно уступают значению предельной прочности силицена при одноосном растяжении.

Таким образом, спаренные листы силицена на графитовой подложке применимы в конструкции анода литий-ионной батареи, в том числе при наличии в силицене дефектов вакансионного типа.

\section{Список литературы}

[1] Y.G. Guo, J.S. Hu, L.J. Wan. Adv. Mater. 20, 2878 (2008).

[2] L. Ji, L. Zhan, M. Alcoutlabi, X. Zhang. Energy Environ. Sci. 4, 2682 (2011).

[3] W. Xu, J. Wang, F. Ding, X. Chen, E. Nasybulin, Y. Zhang, J.G. Zhang. Energy Environ. Sci. 7, 513 (2014).

[4] Y. Xie, Y. Dall'Agnese, M. Naguib, Y. Gogotsi, M.W. Barsoum, H.L. Zhuang, P.R. Kent. ACS Nano 8, 9606 (2014).

[5] W. Li, Y. Yang, G. Zhang, Y.W. Zhang. Nano Lett. 15, 1691 (2015).

[6] H.R. Jiang, Z. Lu, M.C. Wu, F. Ciucci, T.S. Zhao. Nano Energy 23, 97 (2016). 
[7] D. Tsoutsou, E. Xenogiannopoulou, E. Golias, P. Tsipas, A. Dimoulas. Appl. Phys. Lett. 103, 231604 (2013).

[8] L. Meng, Y. Wang, L. Zhang, S. Du, R. Wu, L. Li, Y. Zhang, G. Li, H. Zhou, W.A. Hofer, H.J. Gao. Nano Lett. 13, 685 (2013).

[9] C.L. Lin, R. Arafune, K. Kawahara, M. Kanno, N. Tsukahara, E. Minamitani, Y. Kim, M. Kawai, N. Takagi. Phys. Rev. Lett. 110, 076801 (2013).

[10] S. Cahangirov, M. Audiffred, P. Tang, A. Iacomino, W. Duan, G. Merino, A. Rubio. Phys. Rev. B 88, 035432 (2013).

[11] S. Cahangirov, M. Topsakal, E. Akturk, H. Sahin, S. Ciraci. Phys. Rev. Lett. 102, 236804 (2009).

[12] Y. Cai, C.P. Chuu, C.M. Wei, M.Y. Chou. Phys. Rev. B 88, 245408 (2013).

[13] А.Е. Галашев, К.А. Иваничкина. ЖФХ. 91, 12, 125 (2017).

[14] M. Houssa, A. Molle. J. Phys.: Condens. Matter 27, 253002 (2015).

[15] А.Е. Галашев, К.А. Иваничкина, А.С. Воробьев, О.Р. Рахманова. ФТТ 59, 1218 (2017).

[16] S. Plimpton. J. Comp. Phys. 117, 1 (1995).

[17] J. Tersoff. Phys. Rev. B 38, 9902 (1988).

[18] J. Tersoff. Phys. Rev. B 39, 5566 (1989).

[19] F. Benkabou, M. Certier, H. Aourag. Mol. Sim. 29, 201 (2003).

[20] R. Yu, P. Zhai, G. Li, L. Liu. J. Electron. Mater. 41, 1465 (2012).

[21] S.K. Das, D. Roy, S. Sengupta. J. Phys. F 7, 5 (1977).

[22] T.-E. Fang, J.-H. Wu. Comp. Mater. Sci. 43, 785 (2008).

[23] B. Feng, Z. Ding, S. Meng, Y. Yao, X. He, P. Cheng, L. Chen, K. Wu. Nano Lett. 12, 3507 (2012).

[24] D. Chiappe, C. Grazianetti, G. Tallarida, M. Fanciulli, A. Molle. Adv. Mater. 24, 5088 (2012).

[25] M. Neek-Amal, A. Sadeghi, G.R. Berdiyorov, F.M. Peeters. Appl. Phys. Lett. 103, 261904 (2013).

[26] A.Y. Galashev. Comp. Mater. Sci. 98, 123 (2015).

[27] В.П. Скрипов, А.Е. Галашев. Успехи химии 52, 177 (1983).

[28] R. Roman, S.W. Cranford. Comp. Mater. Sci. 82, 50 (2014).

[29] А.Е. Галашев, Ю.П. Зайков. ЖФХ 89, 12, 1899 (2015).

[30] А.Е. Галашев, Ю.П. Зайков, Р.Г. Владыкин. Электрохимия 52, 1085 (2016).

[31] A.Y. Galashev, K.A. Ivanichkina. Phys. Lett. A 381, 3079 (2017).

[32] А.Е. Галашев, О.Р. Рахманова, Ю.П. Зайков. ФТТ 58, 1786 (2016).

[33] A.Y. Galashev. J. Chem. Phys. 139, 124303 (2013).

Редактор Е.Ю. Флегонтова 